\title{
Research on Mud Pulse MWD Device for Mines
}

\author{
GAO Jun ${ }^{1, *}$ \\ ${ }^{1}$ CCTEG XI'AN Research Institute, Xi'an, Shaanxi, 710077, China
}

\begin{abstract}
As the core accoutrement of directional drilling construction, the measurement while drilling (MWD) device can be divided into three types due to the different data transmission methods: wired, mud pulse and electromagnetic wave. This paper used the mud pulse method to develop a mud pulse MWD device for mines, and the working principle of the mud pulse signal transmission, the signal encoding method and the structure of the device were described. Experimental research showed that the mud pulse wireless MWD device had the advantages of long transmission distance and strong working stability. At the same time, the device was not restricted by the drill pipe during operation, which could be combined with sliding orientation and rotary feed, and had great promotion and application value.
\end{abstract}

\section{Introduction}

Due to its advantages such as controllable trajectory, rotation of hole bottom, and large drilling depth, MWD directional drilling has been used more and more widely in underground gas extraction, water control, geological structure or abnormal body exploration in domestic and foreign coal mines.

As the core accoutrement for measuring while drilling in coal mines, the MWD system for mines is mainly composed of two parts, namely the hole bottom instrument and the orifice instrument. The hole bottom instrument uses a sensor group to measure the inclination, azimuth and tool face angle and then transmits the data to the orifice through the data sending module. The orifice instrument generally uses the mine explosionproof computer to receive the data at the bottom of the hole through the data receiving module, and to calculate, display and store, as shown in Figure 1.

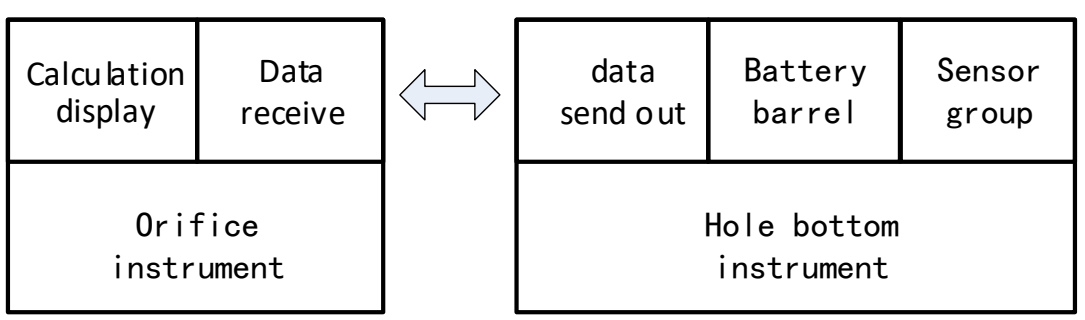

Fig. 1. Composition of MWD system

The data transmission technology in the mine MWD system refers to the data sending and receiving technology between the hole bottom instrument and the orifice instrument, which is the application foundation and key technology of the mine MWD system, and directly affects the construction personnel's ability to timely and accurately obtain the drill bit parameters at the bottom of the hole.

\section{Mud pulse transmission while drilling}

Mud pulse data transmission technology is the most widely used wireless data transmission method in the petroleum field, including three transmission forms: positive pulse, negative pulse and continuous pulse. At present, limited by the gaps in the underground working conditions of coal mining, supporting drilling tools and technological methods, only the mud pulse MWD system that transmits data by positive pulse is adopted in mining products. The positive pulse transmission has the advantages of simple instrument structure, stable signal, and reliable performance, mature technology and other advantages. Its transmission principle is shown in Figure 2. The measured data is encoded in a specific way to generate a pulse signal. The pulse signal is switched through the circuit to control the valve switch to change the flow cross-sectional area of the mud in the drill string. When the valve is open, the mud in the drill string can smoothly pass through the restrictor ring; when the valve is closed, the cross-sectional area of the mud circulation is reduced, thereby generating a positive mud pressure pulse in the drill string. The instrument in the hole controls the time when the valve is opened or closed,

\footnotetext{
$\overline{{ }^{*} \text { Corresponding author: } 281527126 @ q q \text { q.com }}$
} 
thereby controlling the pulse width and interval. The cross-sectional area of the mud flow between the valve and the restrictor ring determines the strength of the signal, that is, the pulse amplitude; the opening and closing time of the valve determines the signal period, that is, the pulse frequency; therefore, we can control the pulse amplitude by selecting the outer diameter of the valve and the inner diameter of the current limiting ring, and control the pulse frequency by selecting the opening and closing time of the valve, so that it can be applied to the working environment of different aperture, different displacement and different hole depths.
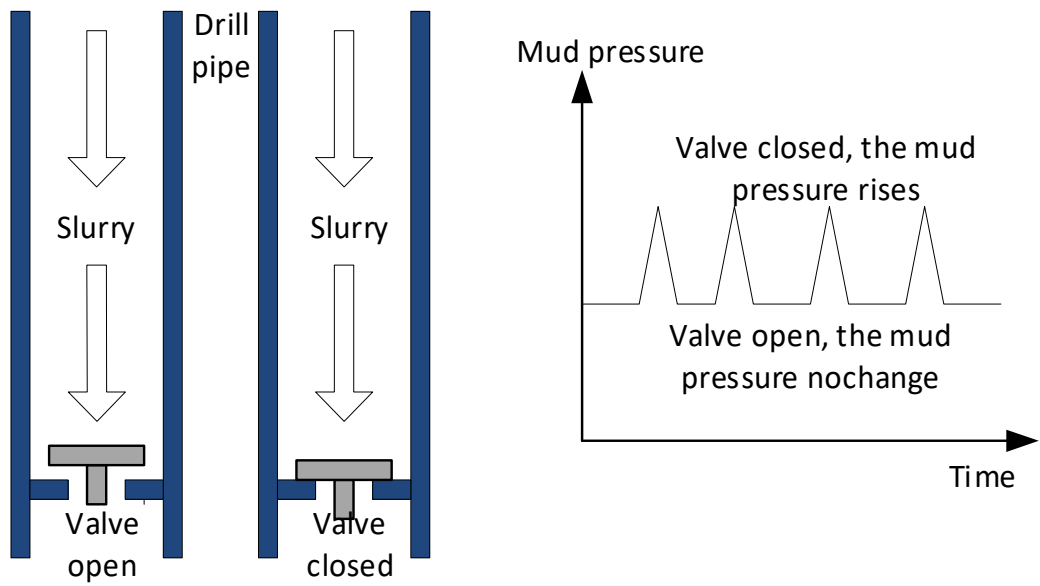

Fig. 2. Mud positive pulse data transmission method

\section{Pulse signal data encoding}

The main purpose of encoding the acquired drilling attitude data is to reduce errors in the pulse signal transmission technology while drilling, and to improve the effectiveness of the overall system. At this stage, with the substantial increase in the amount of downhole data that needs to be collected, and with the increase in the transmission rate of the pulse signal while drilling, the requirements on the encoding and decoding technology will also increase, and the choice of encoding will directly affect the quality of the pulse signal while drilling that is extracted from the ground. At present, the most widely used mud pulse signal data encoding methods in the domestic petroleum industry are Manchester encoding and pulse interval modulation encoding. Manchester encoding can be used in coal mines to satisfy the demand.

Manchester encoding uses rising and falling edges to represent the binary numbers " 0 " and " 1 ". In Manchester encoding, 0 code is the same as clock code and opposite to 1 code, so Manchester encoding is essentially a phase modulation. For example, for binary digital information "00110101", its Manchester encoding schematic diagram is shown in Figure 3.

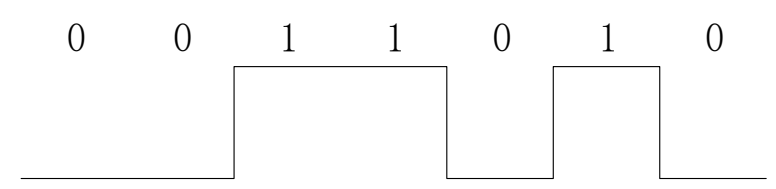

Digital signal

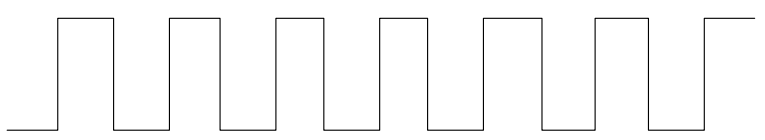

Clock signal

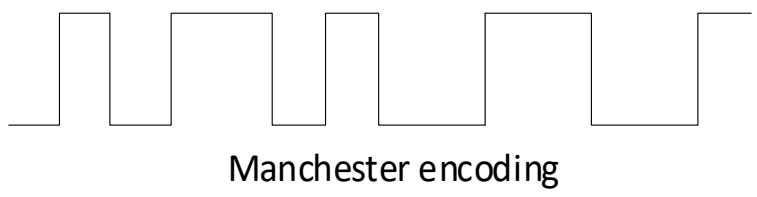

Fig. 3. Schematic diagram of Manchester encoding

According to the clock information, the start and end of the data bit and the width of the bit can be identified.
The Manchester-encoded data stream already contains clock information and data information, so there is no 
need to transmit other redundant information for clock transfer, thereby greatly improving the efficiency of information transmission. The principle of Manchester encoding is simple, and it overcomes the defect that the traditional digital transmission method has no clock, and has certain application value in the process of MWD wireless signal transmission.

\section{Overall design of the device}

Aiming at the special working conditions of coal mines, a full analysis was done of the ransmission characteristics of mud pulse signals in coal mines, and the YHD3-1500 mine mud pulse MWD device was developed, which mainly includes measuring subs, rechargeable battery cylinders, driving subs and pulse generator, etc.

The technical process of the signal transmission of the mine mud pulse MWD device is shown in Figure 1. The device uses rechargeable battery barrels to supply power to the measuring sub and the driving sub respectively. The engineering parameters in the hole such as the measuring angle, azimuth angle and tool face angle of the explosion-proof measuring sub are coded according to Manchester coding protocol, and the measuring data are transmitted to the driving sub through the signal line. The driving sub controls the mud pulse signal generator to close or open the valve, changes the flow area of the drill pipe inner channel, and limits the flow of some clean water into the drill pipe string, so as to generate the mud pressure positive pulse and transfer to the orifice; The explosion-proof pressure transmitter installed at the outlet of mud pump detects the pressure pulse information from the hole, and then transmits it to the explosion-proof computer for processing and display; the explosion-proof pressure loosening device installed at the outlet of mud pump detects the pressure pulse information from the hole and transmits it to the orifice explosion-proof computer for processing and display; after the data transmission is completed, the measuring sub stops working, and the pulse generator restores the channel in the drilling tool to the original area, mud pump pressure returns to normal production, and continues normal drilling.

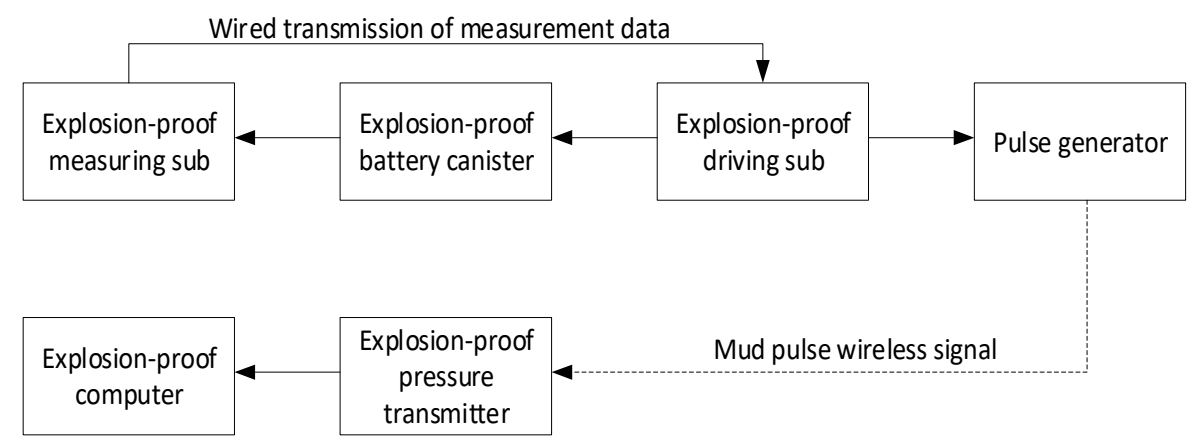

Fig. 4. Flow chart of signal transmission in the device

\section{Conclusion}

The mud pulse data transmission technology uses mud as the communication medium, and uses the pulse of the pressure wave to transmit the bottom data of the hole to the orifice in the form of encoding-decoding. Its own characteristics bring the following two inadequacies on the transmission effect:

(1) Signal interference. The reasons for the interference signal mainly include the mud pump, the amount of air bubbles in the mud, and the influence of movable drilling tools. To reduce the influence of these interference factors, measures should be taken to make the frequency of these interference factors stagger the transmission frequency of the mud pulse signal as much as possible.

(2) Signal retardance. There is a delay time for the pressure signal between the orifice and the hole bottom. On the one hand, the delay time comes from the transmission time of the mud in the drill pipe, that is, the transmission time of the signal pulse in the drill pipe, and on the other hand, it comes from the action time of the valve in the instrument.
In addition, due to the presence of movable mechanisms in the bottom of the hole, the instrument has an impact on the stability of the system. During longterm usage, the state of the pulse valve needs to be checked regularly, and if there is abrasion and deformation, the pulse valve needs to be maintained in time.

\section{Funded Projects}

[1] Development of Explosion-proof Turbine Generator for Underground Wireless While Drilling Measurement (2018XAYMS01).

[2] Major National Science and Technology Special Tasks in the 13th Five-Year Plan

(2016ZX05045-003-001).

\section{References}

1. Tan Chao,Li Zongliao,Wang Jiacheng. Design and Test Analysis of Underground Turbine Generator in Coal Mine[J]. Micromotor,2019,47(05):32-35. 
2. Liu Shuang. Parametric Design of Downhole Generator Turbine while Drilling[D]. China University of Petroleum (East China), 2017.

3. SHI Zhijun,DONG Shuning,YANG Junzhe,Key technology of drilling in-seam directional borehole of $3000 \mathrm{~m}$ in underground coal mine [J]. Coal Geology \& Exploration,2019, 47(6).

4. Jia Huiqin,Wan Mi,Jiao Wei. Design and Experimental Study of Small Diameter Turbogenerator $[\mathrm{J}]$. Electronic Measurement Technology Abroad, 2019, 38(01): 63-66. 